\title{
Das therapeutische Sandspiel als Psychodrama im Sand
}

\author{
Silvia Franke
}

Angenommen: 22. Januar 2021 / Online publiziert: 16. Februar 2021

(C) Der/die Autor(en) 2021

Zusammenfassung Dieser Artikel der ,Zeitschrift für Psychodrama und Soziometrie" beleuchtet das therapeutische Sandspiel aus psychodramatischer Sicht und anhand einer Fallbeschreibung. Es kann positive Spielerinnerungen aktivieren, Kontrolle, Selbstwirksamkeit und gelingende Begegnung ermöglichen und eignet sich aufgrund seiner Ressourcenorientierung speziell für früh traumatisierte KlientInnen, die ihr Innenleben nur schwer verbalisieren können. Aufgrund vieler Parallelen zum Psychodrama kann es als Vorstufe für ein psychodramatisches Rollenspiel dienen.

Schlüsselwörter Psychodrama $\cdot$ Sandspiel $\cdot$ Kreativität $\cdot$ Tele $\cdot$ Trauma

\section{Therapeutic sandplay as psychodrama in the sand}

\begin{abstract}
This article in "Zeitschrift für Psychodrama und Soziometrie" explores therapeutic sandplay from a psychodramatic view and highlights the method based on a case description. It can activate positive psychosomatic play memories to experience control, self efficiacy and rewarding encounters. Due to its resource-orientation it seems especially useful for clients with early traumatic experiences lacking verbal expression for their inner life. Showing many parallels to psychodrama it may act as a preliminary stage for psychodramatic roleplay.
\end{abstract}

Keywords Psychodrama $\cdot$ Sandplay $\cdot$ Creativity $\cdot$ Tele $\cdot$ Trauma

\footnotetext{
S. Franke, Msc ( $\square)$

FH St. Pölten - University of Applied Sciences, Matthias Corvinus-Straße 15, 3100 St. Pölten,

Österreich

E-Mail: silvia.franke@aon.at

Praxis für Psychotherapie, Supervision und Coaching, Erdbergstraße 88/19, 1030 Wien, Österreich
} 
Oft wissen die Hände das Geheimnis zu enträtseln, an dem der Verstand sich vergebens mühte C. G. Jung (1916/1958, S. 99).

\section{Einleitung: Geschichte und Entwicklung des Sandspiels}

Mitte der 1950er-Jahre, als Moreno seine Grundgedanken zur Soziometrie entwickelte, entstand aus der Weiterentwicklung der ,World Technique“ (Lowenfeld 1929) durch die Jung'sche Psychologin und Kindertherapeutin Dora Kalff das therapeutische Sandspiel. Die beiden sind sich zwar nie begegnet (Moreno war zu dieser Zeit schon in den USA und Kalff arbeitete in der Schweiz), doch weisen Psychodrama und Sandspiel Gemeinsamkeiten auf (s. Abs. 5), vor allem durch das Element der Bühne, einer Sandkiste (ca. $72 \times 57 \times 7$ ), in der eine dreidimensionale ,Welt“ in Szene gesetzt werden kann. Als Requisiten stehen Miniaturen aus der realen und Fantasie-Welt (z. B. Menschen, Tiere, Pflanzen, Regenbögen, spirituelle Symbole) zur Verfügung. Themen werden nicht mittels festgelegter Rollen dargestellt, sondern manifestieren sich indirekt und spontan. Mit dieser intuitiven Gestaltung knüpft Kalff an C. G. Jungs Konzept der Entstehung von Träumen als ,unparteiische, der Willkür des Bewusstseins entzogene, spontane Produkte der unbewussten Seele“ an (Jung 1934, S. 32), ähnliches kann auch über die Entstehung von Sandbildern gesagt werden. Nach Lowenfeld geht dem Spracherwerb ein präverbales primäres System kindlichen Denkens voraus, das neben späteren Organisationsstrukturen des Selbst weiterbestehe, es sei nicht rational, gänzlich individuell und könne nicht auf sekundäre Weise, d. h. logisch-verbal ausgedrückt werden, wohl aber in nonverbaler, spontan-kreativer Form wie dem Sandspiel (vgl. Lowenfeld 1948). Dieses wird heute auch bei Erwachsenen eingesetzt und mit anderen Methoden kombiniert, z.B. mit EMDR (Rost 2016), der „Ego-States-Therapie“ (Watkins und Watkins 2003)), dem hypnosystemischen „Narrativen Sandspiel“ (Brächter 2009), weitere Abwandlungen sind das „Sanddrama“ (Toscani 1998), die gruppenzentrierte „Expressive Sandarbeit“ (Pattis Zoja 2012) und das japanische „Hakoniwa“ (Zerbe Enns und Kasai 2003, S. 93-112). Die von mir verwendete Form orientiert sich am Kalff'schen Modell unter Einbezug narrativ-psychodramatischer Elemente.

\section{Ablauf der Sitzung}

Nach dem Vorgespräch nehmen die KlientInnen mit den Händen Kontakt mit dem Sand auf. Manchmal bleibt es bei einem zweckfreien Mäandern der Hände im Sand, meist werden aber Figuren ausgewählt und platziert, dies geschieht intuitiv nach dem Motto „Was mich jetzt in diesem Moment anspricht“. Danach betrachten wir gemeinsam das Bild und ich lasse mir das Geschehen erklären. In Abwandlung der Kalff'schen Form erfrage ich auch, was passieren würde, wenn das Bild in Bewegung käme, dadurch finden fixierte Situationen oft eine stimmige Auflösung. Nach Ende des Spielprozesses wird abseits der „Sandbühne“ das Erlebte gemeinsam besprochen, ohne es zu analysieren, meist erzählen die KlientInnen ohnehin selbst, was ihnen ,aufgegangen“ ist. Das fertige Bild soll als solches belassen und intakt 
im Gedächtnis gespeichert werden, erst am Therapieende werden die von mir dokumentierten Bilder gemeinsam angesehen. Die im Fallbeispiel (Abs. 5) genannte Klientin bestand anfangs darauf, selbst zu fotografieren und alles gemeinsam wegzuräumen (,Ich kann das Bild doch nicht Ihnen überlassen, wer weiß, was Sie damit machen!“). Im Lauf der Zeit veränderte sich das, auch das Fotografieren ließ sie bald sein, was etwas aussagt über die Entwicklung unserer Beziehung.

\section{Rolle der TherapeutInnen, Teleprozess}

Die Funktion der TherapeutInnen beschränkt sich während des Spiels auf die empathische Präsenz im „freien und zugleich geschützten Raum “ (Kalff 2017, S. 9), sie nehmen in einer ,quasi introvertierten Haltung aktiv am Geschehen teil“ (BossBaumann 2006, S. 40). An der Seite der KlientInnen notieren sie die Platzierung der Figuren, achten auf den körperlichen Ausdruck, kommentieren, wenn überhaupt, nur sehr zurückhaltend und unterstützen als anteilnehmende Beobachterin den kreativen Prozess (vgl. Kalff, S. 15). Beide Partner agieren als gleichrangig miteinander Forschende (vgl. Mitchell und Friedman 1997, S. 27-49), das authentische Interesse der TherapeutInnen ähnelt der Freude einer Mutter, die ihr Kind beim Entdecken und Gestalten neuer Welten begleitet. Die sichere therapeutische Beziehung und der überschaubare Rahmen bieten eine verlässliche Basis und ermöglichen eine Balance zwischen Nähe und Exploration (vgl. Ainsworth 2015).

Die Nähe beider Beteiligter im intersubjektiven Raum ist eine stille, intime und bezogene. Kawai (1985, iii-xi) nennt sie „,von Hara zu Hara“ (jap. „Bauch“, „Quelle des Lebens“), diese permanente nonverbale Kommunikation sei der für die Förderung der Aktivität ausschlaggebende heilsame Faktor (vlg. ebd.). Die im Verlauf ungestörten kreativen Schaffens bei den KlientInnen einsetzende limbische Aktivierung betrifft mittels neurophysiologischer Resonanz auch die affektiv teilhabenden TherapeutInnen (vgl. Franke 2011, S. 73 ff.), laut Bradshaw Tauvon (1998, S. 38), entsteht eine „Inter-Psyche“ im Sinne eines wechselseitigen Teleprozesses (vgl. Buer 2004, S. 35). Das Netz telischer Beziehungen verläuft, über die Bande“, d. h. die achtsame Wahrnehmung des Werks der KlientInnen bewirkt bei den TherapeutInnen eine komplexe neuronale Reaktionskette, die zurückwirkt auf die KlientInnen und eine tragfähige Verbindung fördert: „We need the eyes of others to form and hold ourselves together" (Stern 2004, S. 107).

\section{Regression, Nachreifung, geteilte Aufmerksamkeit}

Speziell geeignet ist das Sandspiel bei früh und komplex bindungstraumatisierten KlientInnen mit schwer aushalt- und verbalisierbaren emotionalen oder körperlichen Zuständen. Für sprachliche und emotionale Nachreifung mittels positiver Körpererinnerungen ist die Sandkiste ein ideales Medium: die Kontaktaufnahme mit dem weichen Sand berührt frühe psychosomatische Entwicklungsschichten und das Angenommensein durch die TherapeutInnen ermöglicht einen regressiven, tranceartigen Prozess. Kalff spricht von der Wiederherstellung der ursprünglichen Mutter- 
Kind-Einheit, einer Situation des In-sich-Ruhens, die alle Kräfte zur Persönlichkeitsentwicklung im Keim enthält (vgl. Kalff, S. 15). Für rasch emotional überflutete KlientInnen kann die kontrollierte, entschleunigte Regression im Rahmen einer sicheren Bindung eine heilsame Erfahrung sein, die auch zur Exploration mit anderen Medien ermutigt. Ein weiterer entwicklungsfördernder Aspekt liegt im szenischen Aufbau: KlientInnen und TherapeutInnen befassen sich mit demselben Gegenstand, die TherapeutInnen folgen dem Aufmerksamkeitsfokus der KlientInnen und im abschließenden gemeinsamen Betrachten kommt es zur Einnahme einer Außenperspektive. Die Fähigkeit zur geteilten Aufmerksamkeit (,,joint attention “) wird als notwendige Voraussetzung für die Entwicklung von Sprache und Kognition gesehen (vgl. Tomasello und Farrar 1986, S. 1454-1463). Das Sandspiel kann durch sein Format die gemeinsame „Draufsicht“ und damit sowohl sprachlichen Ausdruck als auch kognitive Distanzierung fördern (interessanterweise finden sich auch im Spiel häufig Figuren, die am Rande das Geschehen betrachten). Die Einnahme einer neutralen Beobachtungsposition ist für traumatisierte KlientInnen ein wichtiger Entwicklungsschritt und wird auch in der Traumatherapie (z. B. EMDR, Bildschirmtechnik) gefördert.

\section{Parallelen und Unterschiede zum Psychodrama}

Sowohl Moreno als auch Jung ging es um die Entwicklung und Manifestation des Selbst durch Kreativität: Moreno wählte mit seiner Betonung der Spontaneität (,When spontaneity grows, the self expands“, Moreno, 1947/2010, S. 23) den expressiven, interpersonellen Weg über das Rollenspiel und die äußere Bühne, Jung einen intrapsychischen durch aktive Imagination und Traumdeutung. Dora Kalff's Sandspiel könnte als Verbindungsstück zwischen den beiden Richtungen betrachtet werden: Wie beim Psychodrama liegt der Schwerpunkt im Spiel selbst, nicht in Diagnostik oder Analyse. Es gibt eine Reihe psychodramatisch wirksamer Elemente: Bühne, Rolleneinkleidung, phasenhafter Verlauf, Förderung der Aktionsfähigkeit, das Entwickeln alternativer Geschichten. Die nicht invasive, haltende Rolle der TherapeutInnen könnte als „stummes Doppeln“ oder „Schutzengelposition“ (vgl. Ottomayer 1992, S. 81) bezeichnet werden. Die Konzeption eines zugrundeliegenden „primären Systems“ findet sich auch in Morenos erstem Universum: „There is a sort or primordial nature which is immortal and returns afresh with every generation, a first universe which contains all beings and in which all events are sacred." (Moreno 1947a, S. 4). Nach Leutz (1974, S. 40) verzögert sich durch frühe Störungen der Übergang vom durch psychosomatisches Erleben geprägten ersten in das zweite Universum, in dem Vorstellung und Realität auseinandergehalten werden können. Hier kann das Sandspiel eine stabilisierende Brückenfunktion einnehmen und den Übergang durch Nachreifung spielerisch erleichtern. Im Sandspiel werden Kompetenzen aller Rollenebenen (Schacht 2003) berührt: psychosomatisch der Kontakt mit dem Element Sand, psychodramatisch das in Szene und Bewegung setzen von Figuren und soziodramatisch der gemeinsame Blick auf deren Konstellation und Beziehungen. Unterschiede finden sich trotz eines monodramaähnlichen Settings im Ablauf (keine vorab zugeordneten Rollen, sehr freie Gestaltung, nur indirekter Bezug zur 
Lage) sowie in der durchwegs zurückhaltenden Rolle der TherapeutInnen. Alles in allem sehe ich aber mehr Gemeinsamkeiten als Unterschiede zwischen Sandspiel und Psychodrama. Die Surplus Reality des Sandspiels kann eine spielerische, wenig angstbesetzte Möglichkeit bieten, seelische Inhalte nach , außen` zu bringen und sie im Rahmen einer greif- und kontrollierbaren Welt gegenständlich zu machen.

\section{Fallbeschreibung}

Aufgrund der Schizophrenieerkrankung der Mutter lebte Frau C. (35) die ersten drei Jahre bei der Großmutter, danach bei einer Pflegefamilie, wo es vom 5.-12. LJ zu sexuellem Missbrauch durch den Pflegevater kam. Mit 16 flüchtete sie in eine Jugendamts-WG und konsumierte Heroin bis zum Beginn einer Substitutionstherapie. Arbeitsversuche hielt sie nie durch, sie ist in Frühpension. Abendmatura und stationäre Therapien scheiterten an Gruppenangst und Dissoziation. Zu Beginn der Therapie litt sie unter Symptomen einer komplexen PTBS wie Dissoziation, Panikattacken und Selbstverletzung. Wegen ihres entgleisten Schlafrhythmus kam sie oft zu spät, Gespräche waren kaum möglich, die Compliance zur Einnahme der psychiatrischen Medikation schwankte. Erst durch das Sandspiel besserten sich die Symptome: sie erscheint pünktlich zur Therapie, kann über sich sprechen und hat sich selbst eine Besuchsbetreuerin durch den Verein LOK („Leben ohne Krankenhaus") organisiert. Den Einsatz imaginativer Therapiemethoden lehnt sie aufgrund ihrer Dissoziationsneigung $\mathrm{ab}$, dafür hat sich das Sandspiel als für sie adäquate Methode erwiesen, sogar eine partielle Traumaexposition mit EMDR war im Verlauf möglich. Die folgenden zwei Sitzungen fanden im ersten Jahr nach Beginn der Sandspieltherapie statt (Übertitel von mir).

\section{2/2019 (,Raus aus dem Ringelspiel“6)}

Frau C. ist heute alles zu viel, sie könne der Unordnung nicht mehr Herr werden und liege meist deprimiert und fernsehend im Bett. In den Sand platziert sie ein Ringelspiel, setzt ein Entchen hinein, darunter einen Frosch, ein Krokodil und einen großen Vogel, der alles beobachtet. Links oben sitzt ein Schmetterling, in der Mitte markieren Steine eine Begrenzung (,Grenzen sind wichtig, es braucht eine Ordnung"), am Rand stecken bunte Federn als Wald, daneben liegt ein langer Pinsel (Malen ist ihr Hobby) (Abb. 1).

Dazu erklärt sie: „Die Ente kann nicht raus dem Ringelspiel, wegen dem Krokodil. Aber das ist eigentlich nicht böse, sondern besorgt, dass der Ente etwas passieren könnte, wenn sie hinaus in die Welt geht. Das kann sie aber eh nicht, weil ihr ganz schwindlig ist vom Ringelspiel, sie würde sich nicht zurechtfinden (Hinweis auf Dissoziation!). Der kleine Frosch hat keine Angst, kann sich überall hinbewegen, setzt sich auf den Pinsel und besucht den Schmetterling“. Als der Vogel dem Krokodil erklärt, dass es hingehen darf, wo es sich wohl fühlt, genießt es seine Freiheit, dafür passt der Vogel auf die Ente auf, die sich in Bewegung setzt. Nun verändert Frau C. die Landschaft: Die Federn werden weiter gesteckt, die Grenze erweitert, damit die Ente Schmetterling und Frosch besuchen kann (Abb. 2). Bei der Nachbesprechung meint Frau C., es gehe ihr 
Abb. 1 Sandbild 1

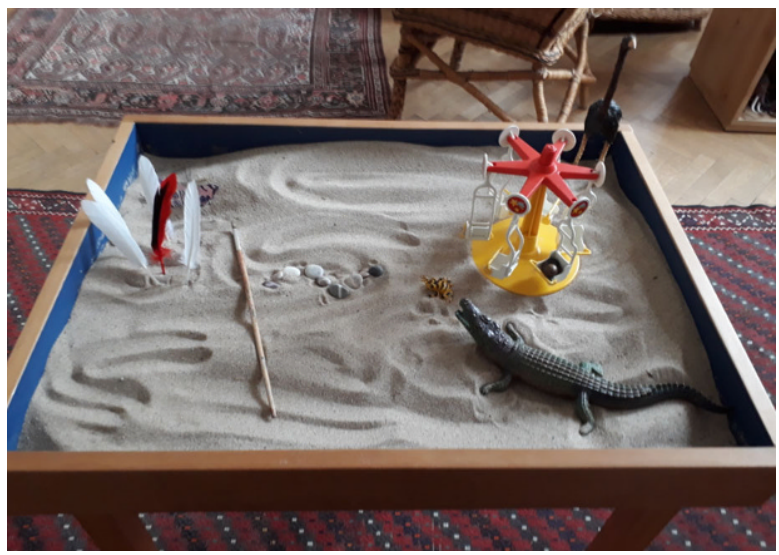

Abb. 2 Sandbild 2

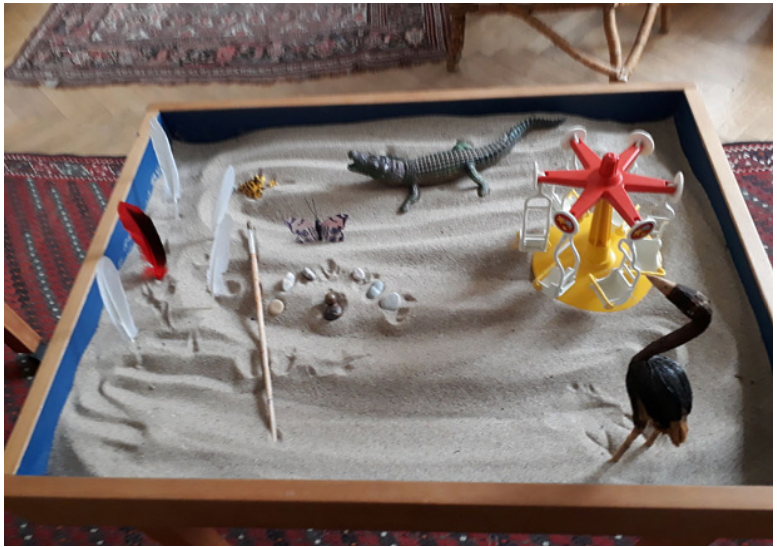

viel besser, es sei ihr klargeworden, dass sie ihre Lage verändern und etwas erleben kann, nicht nur Zuschauerin bleiben muss. Ich erkenne im Bild viele Anteile, die neugierig aufeinander zugehen, einen (das Krokodil), der froh ist, wenn er nicht kontrollieren muss und einen beschützenden (Vogel), der den Überblick behält, eingreift und Veränderung ermöglicht.

\section{4/2019 (,Alle haben es lustig, nur einer sieht $\mathrm{zu}^{6}$ )}

Frau C. weiß heute nicht, was sie will und spürt sich nicht, sie hat Angst vor dem Schreibkurs, für den sie sich angemeldet hat. Im Spiel baut sie mit Tieren aus Bauernhof und Wald, Holzrinden und Bucheckern ein harmonisches Bild auf. Links oben im Eck sitzt ein Bär und ,passt auf, dass da nix passiert“ (Abb. 3).

Die Tiere rühren sich kaum, ,denn sonst wird der Bär sehr nervös“. Dafür bewegt sich aber die Erde, ,,so, wie Sanddünen wandern“. Allen geht es gut, es gibt viele Freundschaften, nur der Bär fühlt sich ausgeschlossen. „Einem geht es immer schlecht in diesen Bildern, da muss man sich drum kümmern! Und immer ist es wer anderer“. Als ich frage, was ihm helfen könnte, setzt sie ihn 
Abb. 3 Sandbild 3

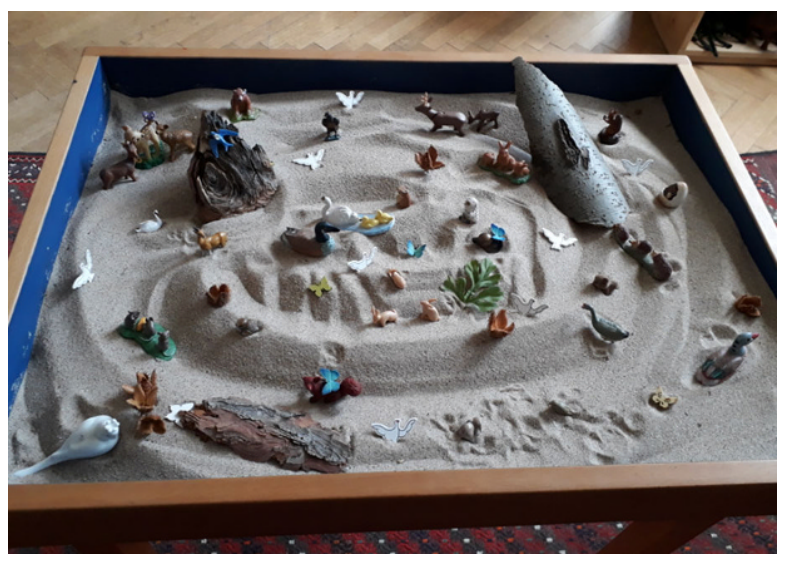

Abb. 4 Sandbild 4

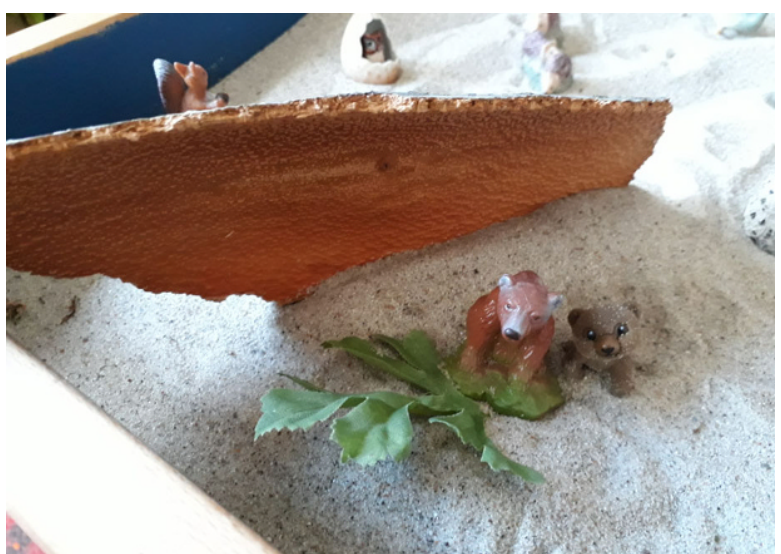

mit einem grünen Blatt (,Er braucht was zu essen“) unter eine Baumrinde, dazu einen Teddybären, so haben die beiden Gesellschaft (Abb. 4). Bei der Nachbesprechung meint sie, sie selbst sei der Bär, der immer nur am Rand zusehe. Er erinnere sie an die Angst, sich in der Schreibgruppe ausgeschlossen zu fühlen. Ich mache sie aufmerksam, dass dort alle mit ihrem eigenen Schreiben beschäftigt sein würden, sie habe dem Bären ja auch ein „Blatt“ mitgegeben, diese Assoziation erheitert sie.

\section{2/2019 (,Zu zweit ist man weniger allein“)}

Frau C. sieht blühend aus, es geht ihr gut trotz reduzierter Substitution, sie freut sich auf den Urlaub mit dem Freund. Die Verliebtheit macht sie froh und auch ängstlich. Zärtlichkeiten erlebe sie als schön, aber es verunsichere sie, dass sie dabei plötzlich in Gefühllosigkeit kippen könne. Auf meine Anregung, einmal feuchten Sand zu probieren, geht sie gern ein. Diesmal arbeitet sie nur mit Sand und formt paarweise Hügel: Schildkröte und Igel, Apfel und Birne, ein Eingangstor, ein sich schlängelnder Wasserlauf spendet allen Teilen Feuchtigkeit und Leben - ein sinnliches, erdverbundenes Bild (das Thema Weiblichkeit 
taucht auch in der nächsten Stunde in Form eines Meermädchens auf). Bei der Nachbesprechung spreche ich die Paarbildungen an. Sie meint nur, Zweisamkeit sei ihr momentan wichtig. Offenbar ist es für sie notwendig, diese neu auftauchende, körperbezogene innere Welt in Ruhe zu belassen. Als ich frage, ob sie das Bild stehen lassen oder gemeinsam wegräumen möchte, schlägt sie mit Lust auf ihr Werk ein (,Das ist das Beste überhaupt!“) und ebnet alles wieder ein.

Psychodramatisch geht die Entwicklung hin zu einem Selbst, das trotz Angst und Unsicherheit aktiv mit anderen in Beziehung treten will. Dies gelingt Frau C. auch im realen Leben immer besser: sie belegte einen zweiten Schreibkurs, eines ihrer Gedichte wird veröffentlicht, sie lässt sich auf die Beziehung zum Freund ein und ist weniger kontrollbedürftig. Neben ihrer Kreativität ist Humor ihre stärkste Ressource, wir lachen oft miteinander über komische Momente im Spiel. Diese Fortschritte wären ohne das (er)lösende Sandspiel nicht möglich gewesen.

Abschließend noch einige ihrer im Schreibkurs zusammengefassten Gedanken zum Sandspiel: „Ich gehe zur Therapie nach Tagen der inneren Anspannung, Depressivität, Angst, Panik, schlechten Erinnerungen, meinem persönlich ausgefochtenen Kampf. Ein freundliches Lächeln empfängt mich, das Gespräch gibt Raum für meine Sorgen. Aus der Dissoziation kann ich trotzdem nicht aussteigen - gefangen in meiner eigenen, vergitterten Welt. Dann auf zur Sandspieltherapie! Erst einmal durch den Sand wühlen, streicheln, drücken, kneten. So verschwindet zumindest ein Teil der Anspannung und ich bin etwas bewusster da. Und ich spüre wieder. Meist arbeiten wir mit Figuren, die ich in der Sandkiste verteile. Meine eigene innere Welt! Nach außen gekehrt, zumindest ein Fragment davon. Ausdrücken, was ich mit Worten alleine nicht sagen kann, mir vor Augen führen, mich befreien. Kurz noch darüber reden. Wie fühlte es sich an, was hat sich getan? Ich gehe oft mit neuen Erkenntnissen, mit weniger Anspannung, mit einem befreiten, guten Gefühl. Danke!“

Funding Open access funding provided by FH St. Pölten - University of Applied Sciences.

Open Access Dieser Artikel wird unter der Creative Commons Namensnennung 4.0 International Lizenz veröffentlicht, welche die Nutzung, Vervielfältigung, Bearbeitung, Verbreitung und Wiedergabe in jeglichem Medium und Format erlaubt, sofern Sie den/die ursprünglichen Autor(en) und die Quelle ordnungsgemäß nennen, einen Link zur Creative Commons Lizenz beifügen und angeben, ob Änderungen vorgenommen wurden.

Die in diesem Artikel enthaltenen Bilder und sonstiges Drittmaterial unterliegen ebenfalls der genannten Creative Commons Lizenz, sofern sich aus der Abbildungslegende nichts anderes ergibt. Sofern das betreffende Material nicht unter der genannten Creative Commons Lizenz steht und die betreffende Handlung nicht nach gesetzlichen Vorschriften erlaubt ist, ist für die oben aufgeführten Weiterverwendungen des Materials die Einwilligung des jeweiligen Rechteinhabers einzuholen.

Weitere Details zur Lizenz entnehmen Sie bitte der Lizenzinformation auf http://creativecommons.org/ licenses/by/4.0/deed.de. 


\section{Literatur}

\section{Verwendete Literatur}

Ainsworth, M. (2015). http://parentalalienationresearch.com/PDF/2015ainsworth.pdf. Zugegriffen: 24. Juli 2020 .

Boss-Baumann, R. (2006). Sandspieltherapie und Spieltherapie. Sandspieltherapie, 20, 40.

Brächter, W. (2009). Narrative Kindertherapie mit der Methode des Sandspiels. Göttingen: Vandenhoeck \& Ruprecht.

Bradshaw Tauvon, K. (1998). Principles of Psychodrama. In M. Karp, P. Holmes \& K. Bradshaw Tauvon (Hrsg.), Handbook of Psychodrama (S. 29-46). London: Routledge.

Buer, F. (2004). Morenos therapeutische Philosophie und die psychodramatische Ethik. In J. Fürst, K. Ottomeyer \& H. Pruckner (Hrsg.), Psychodrama-Therapie (S. 30-58). Wien: Facultas.

Franke, S. (2011). Metaphern der Bezogenheit: Der Begriff des „Tele“ im Psychodrama und seine Parallelen in Psychotherapie, Philosophie und Naturwissenschaft. Masterarbeit. Donauuniversität Krems. http://www.psyonline.at/download/kunden/0015406.pdf. Zugegriffen: 24. Juli 2020.

Jung, C. G. (1934). Die Bedeutung der Psychologie für die Gegenwart. In Wirklichkeit der Seele: Anwendungen und Fortschritte der neueren Psychologie. Zürich: Rascher Verlag.

Jung, C. G. (1916/1958). Die Dynamik des Unbewussten. In: Gesammelte Werke VIII (S. 69). Olten und Freiburg im Breisgau: Walter.

Kalff, D. (2017). Sandspiel. Seine therapeutische Wirkung auf die Psyche (5. Aufl.). München: Ernst Reinhard.

Kawai, H. (1985). Introduction: on transference in Sandplay therapy. In H. Kawai \& Y. Yamanaka (Hrsg.), Studies of sandplay therapy in Japan II (S. iii-xi). Tokyo: Sheisin-Shobo.

Leutz, G. (1974). Das klassische Psychodrama nach J.L. Moreno. Psychodrama: Theorie und Praxis. Bd. 1. Berlin: Springer.

Lowenfeld, M. (1929). http://lowenfeld.org/the-world-technique/. Zugegriffen: 24. Juli 2020.

Lowenfeld, M. (1948). http://lowenfeld.org/book/the-nature-of-the-primary-system/. Zugegriffen: 24. Juli 2020

Mitchell, R., \& Friedman, H. (1997). Konzepte und Anwendungen des Sandspiels. München, Basel: Ernst Reinhardt.

Moreno, J.L. (1947a). The future of man's world (6. Aufl.). New York: Beacon.

Moreno, J. L. (2010). The theatre of spontaneity. UK: North-West Psychodrama Association. Original work Ottomayer, K. (1992). Seminarunterlagen „Psychodrama und Sucht“ (ÖAGG). Unveröffentlicht. S. 81.

Pattis Zoja, E. (2012). Expressive Sandarbeit. Gießen: Psychosozial-Verlag.

Rost, C. (2016). EMDR zwischen Struktur und Kreativität. Paderborn: Junfermann.

Schacht, M. (2003). Spontaneität und Begegnung. Zur Persönlichkeitsentwicklung aus der Sicht des Psychodramas. München: InScenario.

Stern, D. (2004). The present moment in psychotherapy and everyday life. New York: Norton \& Co.

Tomasello, M., \& Farrar, M.J. (1986). Joint attention and early language. Child Development, 57(6), 1454-1463.

Toscani, F. (1998). Sanddrama. Psychodramatic Sandtray with a trauma survivor. The Arts in Psychotherapy, 7(1), 21-30. https://doi.org/10.1016/S0197-4556(97)00058-0.

Watkins, H., \& Watkins, J. (2003). Ego-States - Theorie und Therapie. Heidelberg: Carl Auer.

Zerbe Enns, C., \& Kasai, M. (2003). Hakoniwa: Japanese sandplay therapy. The Counseling Psychologist, 31(1), 93-112. https://doi.org/10.1177/0011000002239403.

\section{Weiterführende Literatur}

Bowlby, J. (1969). http://parentalalienationresearch.com/PDF/1969bowlby.pdf. Zugegriffen: 24. Juli 2020.

Moreno, J. L. (1946). Psychodrama. Bd. 1 (S. xvii). Beacon: Beacon House.

Moreno, J.L. (1954). Die Grundlagen der Soziometrie: Wege zur Neuordnung der Gesellschaft. Opladen: Westdeutscher Verlag.

Moreno, J.L. (1959). Gruppenpsychotherapie und Psychodrama. Einleitung in die Theorie und Praxis. Stuttgart: Thieme. 


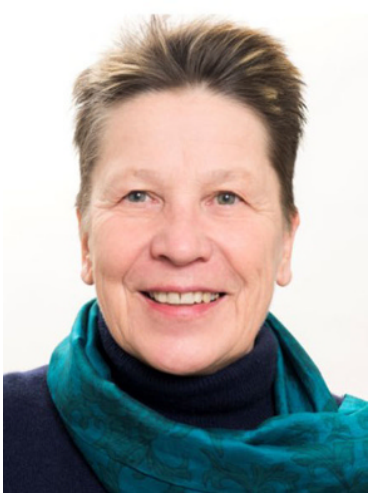

Silvia Franke 1956, DSA, Psychotherapeutin (PD), Dozentin im MALehrgang Suchtberatung und Prävention (FH St. Pölten). ÖAGG, Öst. Netzwerk f. Traumatherapie, EMDR-Netzwerk Österreich. 